\title{
Gravitational wave background from magnetars
}

\author{
T. Regimbau and J. A. de Freitas Pacheco
}

\author{
Observatoire de la Côte d'Azur, Dpt. ARTEMIS and Dpt. CASSIOPEE, BP 4229, 06304 Nice Cedex 4, France \\ e-mail: regimbau@obs-nice.fr
}

Received 27 June 2005 / Accepted 27 September 2005

\begin{abstract}
We investigate the gravitational wave background produced by magnetars. The statistical properties of these highly magnetized stars were derived by population synthesis methods and assumed to be also representative of extragalactic objects. The adopted ellipticity was calculated from relativistic models using equations of state and assumptions concerning the distribution of currents in the neutron star interior. The maximum amplitude occurs around $1.2 \mathrm{kHz}$, corresponding to $\Omega_{\mathrm{gw}} \sim 10^{-9}$ for a type I superconducting neutron star model. The expected signal is a continuous background that could mask the cosmological contribution produced in the early stage of the Universe.
\end{abstract}

Key words. stars: neutron - stars: magnetic fields - gravitational waves

\section{Introduction}

The stochastic background is a rich source of gravitational waves $(\mathrm{GW})$. The detection of a cosmological gravitational background (CGB) is an unique way to probe the very early stages of the universe up to the limits of the Plank era and the big bang (Grishchuk et al. 2001). In addition to the CGB, the emission from a large number of unresolved sources is expected that produces the astrophysical gravitational background (AGB). Because the AGB mask the relic signal, the knowledge of its properties such as the spectral energy distribution is of fundamental importance to determine the best frequency domain in which to search for the CGB in order to optimize the subtraction techniques. A large number of astrophysical processes able to generate a stochastic background have been investigated. On the one hand, distorted black holes (Ferrari et al. 1999a; de Araújo et al. 2000) and bar mode emission from young neutron stars (Regimbau 2001) are examples of sources able to generate a shot noise signal (the time interval between events is large in comparison to the duration of a single event), while supernovas (Blair et al. 1997; Coward et al. 2000; Buonanno et al. 2004) are expected to produce an intermediate "popcorn" noise. On the other hand, the contribution of tri-axial rotating neutron stars results in a truly continuous background that may compete with the relic one in the frequency domain of ground based interferometers (Regimbau \& de Freitas Pacheco 2001b, hereafter RP01b). Neutron stars (NS) are one of the most promising sources for observation by ground based gravitational wave interferometers such as LIGO, VIRGO, GEO and TAMA (see de Freitas Pacheco 2001, for a recent review). Various processes related to NS able to produce large numbers of gravitational waves (GWs) have been investigated: free precession of an axisymmetric star induced by a misalignment between the spin and the symmetry axes (Zimmermann \& Szedenits 1979), r- or bar-mode instabilities excited by the so-called CFS (Chandrasekhar-Friedman-Schultz) mechanism, which may occur in fast and hot rotating neutron stars (Andersson \& Kokkotas 2001, and references therein) and mini gravitational collapse induced by a phase transition in the core (Marranghello et al. 2002). However, the emission produced by a tri-axial rotating star is still one of the most studied mechanisms. Different scenarios leading to a distorted star have been discussed in the literature, as anisotropic stresses from strong magnetic fields and tilting of the symmetry axis during the initial cooling phase when the crust solidifies. Bildsten (1998) pointed out that a neutron star in a state of accretion may develop non-axisymmetric temperature variations on the surface, which produce horizontal density patterns able to create a large mass quadrupole moment, if the elastic response of the crust is neglected. More detailed calculations (Ushomirsky et al. 2000) indicate that the inclusion of the crustal elasticity decreases the expected mass quadrupole by a factor of 20-50, reducing considerably the predicted GW emission. More recently, elastic deformations of compact objects with "exotic" equations of state have been considered by Owen (2005). He showed that solid strange stars could sustain ellipticities as high as few times $10^{-4}$, considerably higher than estimated values for conventional neutron stars (Thorne 1980). The distortion induced by magnetic fields becomes significant in highly magnetized neutron stars, overwhelming the "flattening" due to a fast rotation. The existence of neutron stars with magnetic fields in excess of $10^{14} \mathrm{G}$ (called magnetars) was firstly suggested by Thompson \& Duncan (1992). They have shown that in fast newborn neutron stars, the dynamo mechanism could generate magnetic field strengths up to $10^{16} \mathrm{G}$. The existence of magnetars is supported by the observation of "soft gamma 
repeaters" (SGRs) and anomalous X-ray pulsars (AXPs), whose rotation periods and deceleration rates, if interpreted in terms of the canonical magnetic dipole model, suggest that these objects are associated with young highly magnetized NS (Kouveliotou et al. 1998, 1999; de Freitas Pacheco 1998; Heyl \& Kulkarny 1998; Mereghetti 1999). On the one hand, magnetars may be considered as objects having an origin and evolutionary path different from "classical" radio-pulsars but, on the other hand, simulations based on population synthesis methods suggest that NS are born with a large variety of rotational periods, magnetic fields and that magnetars are objects simply born in the high end of the magnetic field distribution (Regimbau \& de Freitas Pacheco 2001a, hereafter RP01a). According to these simulations, a non-negligible fraction of NS are born with magnetic fields higher than $10^{14} \mathrm{G}$, raising the possibility that these objects may also generate a continuous stochastic background. In RP01b, the contribution of tri-axial rotating neutron stars to the gravitational wave background was calculated under the assumption that the initial period and magnetic field distributions derived from population synthesis of galactic pulsars by Regimbau \& de Freitas Pacheco (2000, hereafter RP00) could be extended to the extragalactic population. In this paper, we adopt a similar procedure to compute the contribution of magnetars to the GW background, but with a fundamental difference. In RP00 the equatorial ellipticity was taken as a free parameter, whereas in the present work the equatorial ellipticity is estimated from NS models including different magnetic field configurations and equations of state. This paper is organized as follows: in Sect. 2 we discuss the properties of the magnetar population, including the magnetic field distribution and the equatorial deformation, in Sect. 3 their contribution to the gravitational wave background is calculated, in Sect. 4 the expected signal is compared to the CGBs and the detection possibility by present and future generations of GW detectors is discussed and in Sect. 5 the main conclusions are summarized.

\section{Properties of magnetars}

\subsection{The magnetic field distribution}

In our previous work (RP00), the population synthesis method was used to recover the statistical properties of the real population of radio pulsars, since most of the objects are hidden by strong selection effects, such as the flux density limit of radio telescopes and their orientation with respect to the pulsar emission beam. These simulations were recently upgraded in order to take into account the new pulsars discovered by the Parkes multibeam survey. In agreement with our previous analysis, the initial period follows a normal distribution of mean $240 \mathrm{~ms}$ with a dispersion of $80 \mathrm{~ms}$, while the magnetic field has lognormal distribution of mean $\log B=13.0$ (in Gauss) with a dispersion of $\sigma_{\log B}=0.8$. For details of these simulations, the reader is referred to RP00.

As shown in Fig. 1, the magnetic field distribution of the observed population matches quite well that derived from the observed period deceleration rate and the "canonical" magnetic dipole model. In this case, the mean field of the observed population is about $1.6 \times 10^{12} \mathrm{G}$ and the distribution has an

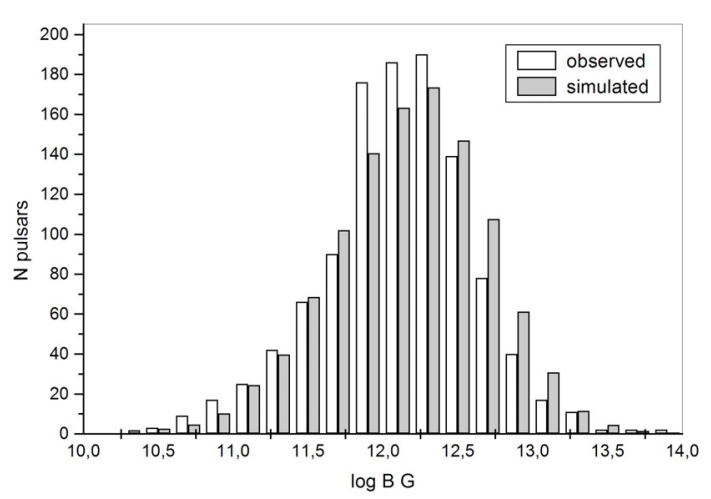

Fig. 1. Comparison between the simulated and the observed distribution (derived from the "canonical" magnetic dipole model) of the magnetic field (in Gauss).

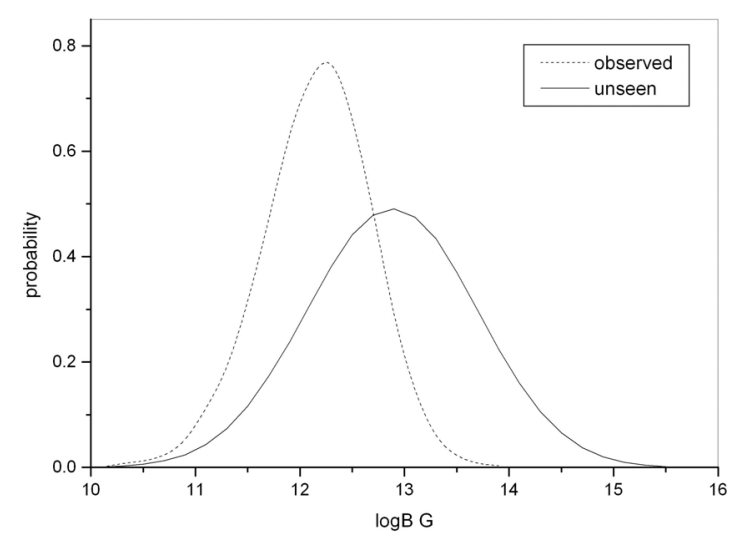

Fig. 2. Magnetic field distributions of the observed (dashed line) and the unseen population (full line) of pulsars.

apparent cutoff around $B=10^{14} \mathrm{G}$. As we have already stated (RP01a), the average magnetic field of the real (or "hidden") population is one order of magnitude higher and the fraction of magnetars, defined as objects with fields above $10^{14} \mathrm{G}$, is around $8 \%$. A comparison between the magnetic field distributions of the observed and the "unseen" populations is given in Fig. 2. Notice that objects with fields as high as $3 \times 10^{15}$ are expected to exist in the Galaxy.

\subsection{Deformation by magnetic fields}

The deformation of magnetized Newtonian stars was already discussed in the early fifties by Chandrasekhar \& Fermi (1953) and Ferraro (1954). The gravitational wave emission from magnetic distorted stars was considered by Gal'tsov \& Tsvetkov (1984), Bonazzola \& Gourgoulhon (1996), Konno et al. (1999, 2000), Palomba (2001), among others. The deformation of a slowly rotating magnetized star can be expressed as the sum of the contribution of three main terms: the first and the most important corresponds to the Lorentz force, induced by current flows in the highly conductive NS interior; the second term represents variations of the gravitational potential, a consequence of the distortion itself and the third is a purely relativistic term arising from the definition of the circumferential radius $R=L /(2 \pi)$, where $L$ is the length of the equator as 


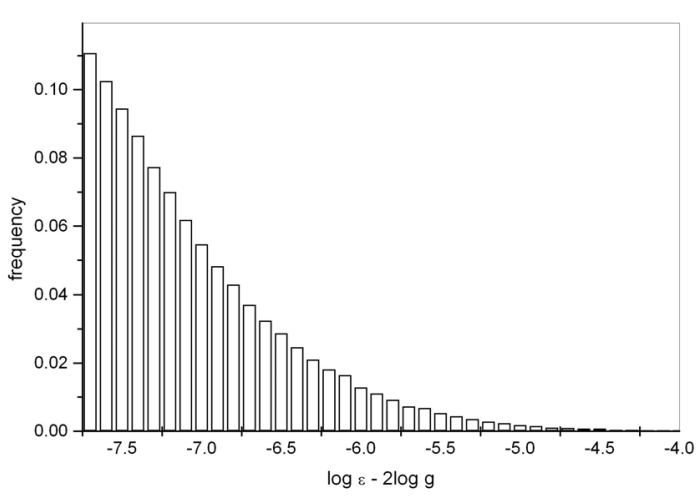

Fig. 3. Simulated distribution probability for the expected magnetic ellipticity of magnetars.

measured by a non-rotating observer. In general, the ellipticity induced by magnetic field effects can be expressed by the dimensionless ratio (Konno et al. 2000)

$\varepsilon_{B}=g \frac{B^{2} R^{4}}{G M^{2}} \sin ^{2} \alpha=1.9 \times 10^{-8} g B_{14}^{2} R_{10}^{4} M_{1.4}^{-2} \sin ^{2} \alpha$

where $B, R$ and $M$ are respectively the magnetic field at the surface, the radius and the mass of the star, $\alpha$ is the angle between the spin and magnetic dipole axes, while $g$ is a dimensionless parameter depending on both the equation of state (EOS) and on the magnetic field geometry. It is equivalent to the parameter $\beta$ introduced by Bonazzola \& Gourgoulhon (1996).

In the case of an incompressible fluid star with a dipole magnetic field, considered by Ferraro (1954), the deformation parameter is $g=12.5$. Relativistic models based on a polytropic EOS, e.g., $P \propto \rho^{\Gamma}$ and a dipole field geometry give similar values (Konno et al. 2000). NS models built with the EOS UV UN $_{14}+$ TNI (Wiringa et al. 1988) and with nonsuperconducting neutron star matter lead to deformation parameters $g$ of the order of the unity for currents concentrated in the crust and a few times higher if currents are concentrated in the core (Bonazzola \& Gourgoulhon 1996). These values correspond to ellipticities of about $(2-20) \times 10^{-8}$ for fields typically of the order of $10^{14} \mathrm{G}$, below the limits estimated by RP01b to produce a detectable signal by the present generation of interferometric detectors. A more favourable situation occurs if neutron stars have a superconducting interior of type I. In this case, the magnetic field permeates only the very outer layers of the star and distortion parameters as high as $g \approx 520$ can be obtained (Bonazzola \& Gourgoulhon 1996). From Eq. (1) and the magnetic field distribution of the actual population derived in the previous section, the ellipticity distribution of magnetars can be calculated (Fig. 3).

Our simulated data can be quite well fitted by the function

$p(x)=2.36 \exp \left(-0.2(x+9.7)^{2}\right)$ if $x>-7.7$

where $x=\log \left(\varepsilon_{B}\right)-2 \log (g)$.

According to our simulations, even if magnetars with ellipticities as high as $\varepsilon_{B} \sim 0.01$ are expected to exist, their gravitational radiation remains small, since most of them have low rotation frequencies (RP01a). This implies that in spite of their high ellipticity, these objects are always decelerated by the

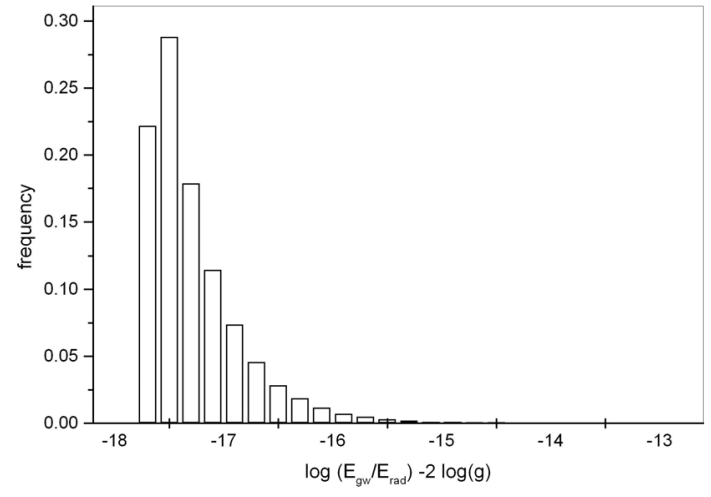

Fig. 4. The ratio between the gravitational and magnetic energy loss rates for magnetars.

magnetic braking mechanism, a point to be retained when the spectral energy is computed (see Sect. 3). To illustrate this point, the distribution of the ratio $R$ between the two energy loss mechanisms, namely

$R=\frac{\dot{E}_{\mathrm{gw}}}{\dot{E}_{\mathrm{md}}}=1.36 \times 10^{-12}\left(\frac{g}{13}\right)^{2} B_{14}^{2} \sin ^{2} \alpha P^{-2}$

is shown in Fig. 4. The numerical value was calculated for a "canonical" NS of mass $M=1.4 M_{\odot}$, radius $R=10 \mathrm{~km}$, moment of inertia $I=1.4 \times 10^{45} \mathrm{~g} \mathrm{~cm}^{2}$ and $B=10^{14} \mathrm{G}$. Notice that even for "extreme" cases, e.g., magnetars in the tail of the period and magnetic field distributions, the aforementioned ratio is $R \sim 0.002 \ll 1$ for objects with $P \sim 1 \mathrm{~ms}, B_{14} \sim 1$ and $g \sim 520$.

\section{The stochastic gravitational wave background}

\subsection{Spectral properties}

The superposition of the gravitational wave emission from different objects and in particular from magnetars formed since the beginning of the star formation in the universe produces a stochastic background whose detection might be possible by employing cross correlation techniques between two antennas (Grishchuk 1976; Christensen 1997).

The spectral properties of the stochastic background are characterized by the dimensionless parameter (Ferrari et al. 1999):

$\Omega_{\mathrm{gw}}\left(v_{\mathrm{o}}\right)=\frac{1}{c^{3} \rho_{\mathrm{c}}} v_{\mathrm{o}} F_{v_{\mathrm{o}}}$

where $v_{\mathrm{o}}$ is the wave frequency in the observer frame, $\rho_{\mathrm{c}}$ is the critical mass density needed to close the Universe, related to the Hubble parameter $H_{0}$ by

$\rho_{\mathrm{c}}=\frac{3 H_{0}^{2}}{8 \pi G}$.

$F_{v_{\mathrm{o}}}$ is the gravitational wave flux at the observer frequency $v_{\mathrm{o}}$ integrated over all sources defined by

$F_{v_{\mathrm{o}}}=\int_{0}^{z_{\max }} f_{v_{\mathrm{o}}} \mathrm{d} R(z)$ 
where $f_{v_{\mathrm{o}}}$ is the gravitational flux of a single source located between $z, z+\mathrm{d} z$ and $\mathrm{d} R(z)$ is the source formation rate. Both quantities are given by

$f_{v_{\mathrm{o}}}=\frac{1}{4 \pi d_{L}^{2}} \frac{\mathrm{d} E_{\mathrm{gw}}}{\mathrm{d} v}(1+z)$

and

$\mathrm{d} R(z)=\lambda_{\mathrm{p}} \frac{R_{\mathrm{c}}(z)}{1+z} \frac{\mathrm{d} V}{\mathrm{~d} z} \mathrm{~d} z$.

In the above equations, $d_{L}=(1+z) r$ is the distance luminosity, $r$ is the proper distance, which depends on the adopted cosmology, $\mathrm{d} E_{\mathrm{gw}} / \mathrm{d} v$ is the gravitational spectral energy emitted by a single source and $v=(1+z) v_{\mathrm{o}}$ the frequency in the source frame. $R_{\mathrm{c}}(z)$ is the cosmic star formation rate and $\lambda_{\mathrm{p}}$ is the mass fraction converted into neutron star progenitors with magnetic fields higher than $10^{14} \mathrm{G}$. The $(1+z)$ term in the denominator of Eq. (8) corrects the star formation rate by the time dilatation due to the cosmic expansion. The element of comoving volume is given by

$\mathrm{d} V=4 \pi r^{2} \frac{c}{H_{0}} \frac{\mathrm{d} z}{E\left(\Omega_{i}, z\right)}$

with

$E\left(\Omega_{i}, z\right)=\left[\Omega_{\mathrm{m}}(1+z)^{3}+\Omega_{\mathrm{v}}\right]^{1 / 2}$

where $\Omega_{\mathrm{m}}$ and $\Omega_{\mathrm{v}}$ are respectively the present values of the density parameters due to matter (baryonic and non-baryonic) and the vacuum, corresponding to a non-zero cosmological constant. A "flat" cosmological model $\left(\Omega_{\mathrm{m}}+\Omega_{\mathrm{v}}=1\right)$ was assumed. Porciani \& Madau (2001) provide three models for the cosmic star formation rate (SFR) history up to redshifts $z \sim 5$. Differences between these models are mainly due to the various corrections applied, in particular those due to extinction by the cosmic dust. In our computations, the three possibilities were considered. Since the final results are not significantly different according to the adopted SFR history, we present here only results derived from the second model, labelled SFR2.

In our calculation, we have taken $\Omega_{\mathrm{m}}=0.30$ and $\Omega_{\mathrm{v}}=$ 0.70 , corresponding to the so-called concordant model derived from observations of distant type Ia supernovae (Perlmutter et al. 1999; Schmidt et al. 1998) and the power spectra of the cosmic microwave background fluctuations (Spergel et al. 2003). The Hubble parameter $H_{0}$ was taken to be $65 \mathrm{~km} \mathrm{~s}^{-1} \mathrm{Mpc}^{-1}$.

Combining the equations above one obtains:

$\Omega_{\mathrm{gw}}\left(v_{\mathrm{o}}\right)=\frac{8 \pi G}{3 c^{2} H_{0}^{3}} \lambda_{\mathrm{p}} v_{\mathrm{o}} \int_{0}^{z_{\text {sup }}} \frac{\mathrm{d} E_{\mathrm{gw}}}{\mathrm{d} v} \frac{R_{\mathrm{SFR} 2}(z)}{(1+z)^{7 / 2}} \mathrm{~d} z$

or numerically:

$\Omega_{\mathrm{gw}}\left(v_{\mathrm{o}}\right)=7.5 \times 10^{-56} h_{65}^{-2} \lambda_{\mathrm{p}} v_{\mathrm{o}} \int_{0}^{z_{\text {sup }}} \frac{\mathrm{d} E_{\mathrm{gw}}}{\mathrm{d} v} \frac{R_{\mathrm{SFR} 2}(z)}{(1+z)^{7 / 2}} \mathrm{~d} z$.

In the above equation, the SFR (in $M_{\odot} \mathrm{Mpc}^{-3} \mathrm{yr}^{-1}$ ) as given by Porciani \& Madau (2001) was derived for a matter dominated cosmology $\left(\Omega_{\mathrm{m}}=1\right)$ with $H_{0}=65 \mathrm{~km} \mathrm{~s}^{-1} \mathrm{Mpc}^{-1}$, but Eqs. (11) and (12) already include the correction for the adopted cosmology. The upper limit of the integral is determined by the frequency cutoff in the source frame, namely

$z_{\text {sup }}=\frac{v_{\text {sup }}}{v_{\mathrm{o}}}-1$.

Here we have assumed that the minimum rotation period is $\sim 0.8 \mathrm{~ms}$, which corresponds to a maximum gravitational wave frequency $v_{\text {sup }} \approx 2500 \mathrm{~Hz}$. The cosmic SFR is quite uncertain for $z>5$, but if one imposes the restriction $z_{\text {sup }} \sim 5$, there are no practical consequences since objects formed at higher redshifts do not contribute significantly to the integral in Eq. (12).

\subsection{Magnetar parameters}

The mass fraction of formed stars giving origin to magnetars is $\lambda_{\mathrm{p}}=\chi \int_{10}^{40} \xi(m) \mathrm{d} m$, where $\xi(m) \propto m^{-2.35}$ is the initial mass function, supposed to be given by Salpeter's law, and NS progenitors are supposed to form in the mass range $10-40 M_{\odot} \cdot \chi$ is the fraction of NS born with magnetic fields $B \geq 10^{14} \mathrm{G}$, which from our simulations is about $8 \%$. Thus, $\lambda_{\mathrm{p}}=3.9 \times 10^{-4} M_{\odot}^{-1}$.

The total spectral gravitational energy emitted by a rotating neutron star is given by

$\frac{\mathrm{d} E_{\mathrm{gw}}}{\mathrm{d} v}=\frac{\mathrm{d} E_{\mathrm{gw}}}{\mathrm{d} t}\left|\frac{\mathrm{d} t}{\mathrm{~d} v}\right|$

where the frequency evolution, according to the analysis of Sect. 2.2, is controlled by the magnetic breaking. In this case, one obtains (RP01b)

$\frac{\mathrm{d} E_{\mathrm{gw}}}{\mathrm{d} \nu}=K \nu^{3}=\frac{512 G}{5 c^{5}} \pi^{6} \varepsilon^{2} I_{z z}^{2} \frac{\tau_{0}}{P_{0}^{2}} \nu^{3}$.

Notice that here we have corrected the above equation by the factor of two missing in the original equation derived by RP01b. The ratio between the initial period $P_{0}$ and the magnetic braking time-scale $\tau_{0}$ is

$\frac{P_{0}^{2}}{\tau_{0}}=\frac{4 \pi^{2} R^{6}}{3 I_{z z} c^{3}} B^{2}$.

For magnetars, the ellipticity is given by Eq. (1) and in this case the constant $K$ is given by

$K=\frac{384 \pi^{4}}{5} g^{2} I_{z z}^{3}\left(\frac{R}{G^{1 / 2} M^{2} c}\right)^{2} B^{2} \sin ^{4}(\alpha)$

or, numerically

$K=2.0 \times 10^{31} g^{2} I_{45}^{3} R_{10}^{2} M_{1.4}^{-4} B_{14}^{2} \sin ^{4}(\alpha)$

where $I_{z z}$ is given in units of $10^{45} \mathrm{~g} \mathrm{~cm}^{2}$.

According to our simulations, the average value of the quantity $\left\langle\left(B_{14}^{2} \sin ^{4} \alpha\right)\right\rangle$ for the galactic population is 32.2 , which will be assumed to be also representative of extra-galactic magnetars. Notice that if the migration of the magnetic dipole is introduced as in RP01, the average $\left\langle\left(B_{14}^{2} \sin ^{4} \alpha\right)\right\rangle$ should be replaced by $\left\langle\left(B_{14}^{2} \sin ^{2} \alpha\right)\right\rangle$ in Eq. (17). However, this alternative model does not change significantly the numerical value of $K$, since the migration scale time is rather short $\left(<10^{4} \mathrm{yr}\right)$ and, as a consequence, most of the objects will be quickly found in an orthogonal configuration. 


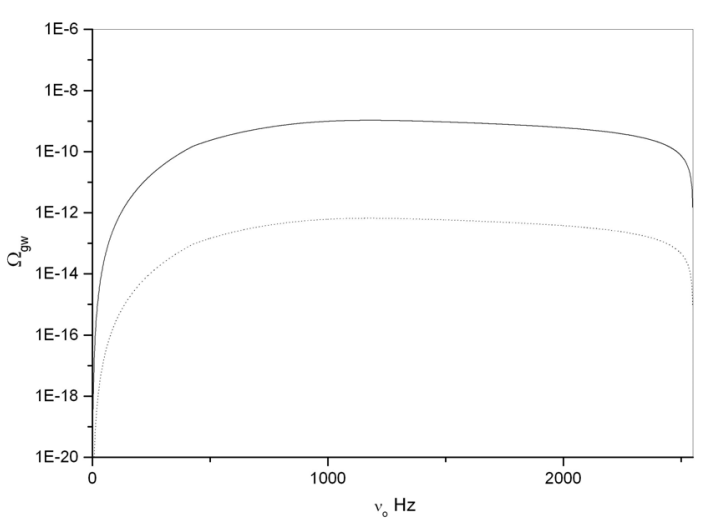

Fig. 5. Density parameter $\Omega_{\mathrm{gw}}$ for the stochastic background produced by magnetars for the two models considered in this work. Model A, solid curve and model $\mathrm{B}$, dotted curve.

\section{Results and detectability}

Figure 5 shows the density parameter $\Omega_{\mathrm{gw}}$ as a function of the observed frequency for two NS models. Model A considers a normal interior (non-superconducting), having a representative deformation parameter $g=13$ (Konno et al. 2000); model B assumes the existence of a type I superconducting interior, characterized by a deformation parameter $g=520$ (Bonazzola \& Gourgoulhon 1996).

The density parameter $\Omega_{\mathrm{gw}}$ reaches a maximum around $1200 \mathrm{~Hz}$ and has a high frequency cut-off at $\sim 2500 \mathrm{~Hz}$. The maximum value of the density parameter is about $6 \times 10^{-13}$ in the case of model A and $\sim 10^{-9}$ if the NS has a superconducting interior.

If $\bar{\tau}$ is the duration of the signal produced by a single source, the stochastic background from magnetars is expected to be continuous since the duty cycle satisfies $D=\int_{0}^{\infty} \mathrm{d} R(z) \bar{\tau}(1+$ $z) \gg 1$. Because it obeys Gaussian statistics and can be confounded with the instrumental noise background of a single detector, the optimal detection strategy is to cross correlate the output of two (or more) detectors, assumed to have independent spectral noises. The cross correlation product is given by (Allen \& Romano 1999):

$Y=\int_{-\infty}^{\infty} \tilde{s_{1}^{*}}(f) \tilde{Q}(f) \tilde{s_{2}}(f) \mathrm{d} f$

where

$\tilde{Q}(f) \propto \frac{\Gamma(f) \Omega_{\mathrm{gw}}(f)}{f^{3} P_{1}(f) P_{2}(f)}$

is a filter that maximizes the signal to noise ratio $(S / R)$. In the above equation, $P_{1}(f)$ and $P_{2}(f)$ are the power spectral noise densities of the two detectors and $\Gamma$ is the non-normalized overlap reduction function, characterizing the loss of sensitivity due to the separation and the relative orientation of the detectors. The optimized $S / N$ ratio for an integration time $T$ is given by (Allen 1997):

$\left(\frac{S}{N}\right)^{2}=\frac{9 H_{0}^{4}}{8 \pi^{4}} T \int_{0}^{\infty} \mathrm{d} f \frac{\Gamma^{2}(f) \Omega_{\mathrm{gw}}^{2}(f)}{F^{2} f^{6} P_{1}(f) P_{2}(f)}$.
Table 1. Expected $\Omega_{\min }$ for the main detector pairs, corresponding to a flat background spectrum and an integration time $T=1$ year, a detection rate $\alpha=90 \%$ and a false alarm rate $\gamma=10 \%$. LHO and LLO stand for LIGO Hanford Observatory and LIGO Livingston Observatory.

\begin{tabular}{lcccc}
\hline \hline & LHO-LHO & LHO-LLO & LLO-VIRGO & VIRGO-GEO \\
\hline Initial & $4 \times 10^{-7}$ & $4 \times 10^{-6}$ & $8 \times 10^{-6}$ & $8 \times 10^{-6}$ \\
Advanced & $6 \times 10^{-9}$ & $1 \times 10^{-9}$ & & \\
\hline
\end{tabular}

In the literature, the sensitivity of detector pairs is usually given in terms of the minimum detectable amplitude for a flat spectrum $\left(\Omega_{\mathrm{gw}}\right.$ equal to constant) (Allen \& Romano 1999)

$$
\begin{aligned}
\Omega_{\min }= & \frac{4 \pi^{2}}{3 H_{0}^{2} T}\left(\operatorname{erfc}^{-1}(2 \alpha)-\operatorname{erfc}^{-1}(2 \gamma)\right) \\
& \times\left[\int_{0}^{\infty} \mathrm{d} f \frac{\Gamma^{2}(f)}{f^{6} P_{1}(f) P_{2}(f)}\right]^{-1 / 2}
\end{aligned}
$$

The expected minimum detectable amplitudes for the main detector pairs, after one year integration, are given in Table 1, for a detection rate $\alpha=90 \%$ and a false alarm rate $\gamma=10 \%$.

The power spectral densities used for the present calculation can be fond in Damour et al. (2001).

$\Omega_{\min }$ is of the order of $10^{-6}-10^{-5}$ for the first generation of interferometers combined as LIGO/LIGO and LIGO/VIRGO. Their advanced counterparts will permit an increase of two or even three orders of magnitude in sensitivity $\left(\Omega_{\min } \sim\right.$ $10^{-9}-10^{-8}$ ). The pair formed by the co-located and co-aligned LIGO Hanford detectors, for which the overlap reduction function is equal to one, is potentially one order of magnitude more sensitive than the Hanford/Livingston pair, if instrumental and environmental noises can be removed. However, the spectrum of magnetars is not flat and the maximum occurs out of the optimal frequency band of ground based interferometers, which is typically around few hundreds Hz, as in Fig. 6. Considering the co-located and co-aligned advanced LIGO interferometer pair, we find a signal-to-noise ratio $S / N \sim 3 \times 10^{-6}$ and $\sim 5 \times 10^{-3}$ for models $\mathrm{A}$ and $\mathrm{B}$ respectively. A possible solution to increase the sensitivity at higher frequencies would be to use the interferometers in a narrowband (rather than in a wideband) configuration, as has been proposed for GEO 600, with the frequency of maximal sensitivity centred around $1 \mathrm{kHz}$. This could be achieved by choosing a high (rather than low) reflectivity of the signal in the recycling mirror, which would concentrate the power in a narrow band rather than distribute it in a wideband. The resonance frequency of the recycling cavity is tunable to the desired frequency by shifting the signal-recycling mirror.

Unless the deformation parameter $g$ is substantially higher than the present expectations, our results indicate that its contribution to the gravitational background is not detectable by the present generation antennas and also probably by their advanced counterparts. A detection could be possible if the interior of the NS is constituted by a type II superconductor. Effective quark-quark interactions in the deconfined core may form a diquark condensate, which behaves as a superconductor of the second kind (Blaschke et al. 1999), able to develop quite 


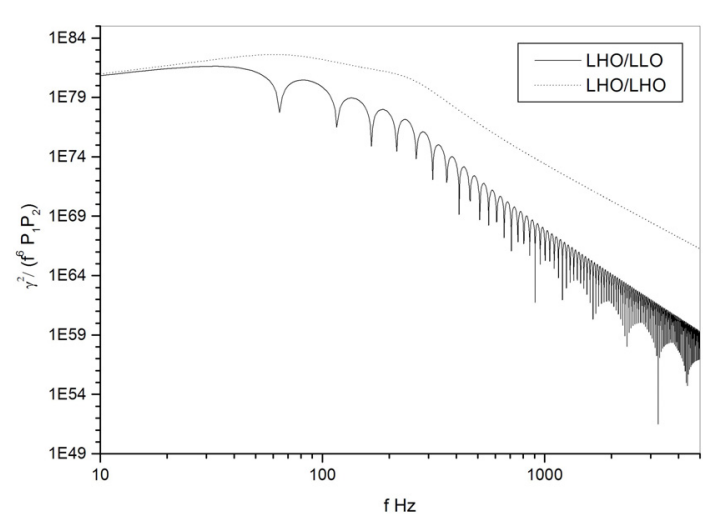

Fig. 6. Integrand of the signal to noise ratio (see Eq. (21)) for advanced LIGO pairs: LHO-LHO (continuous line) and LHO-LLO (dot line).

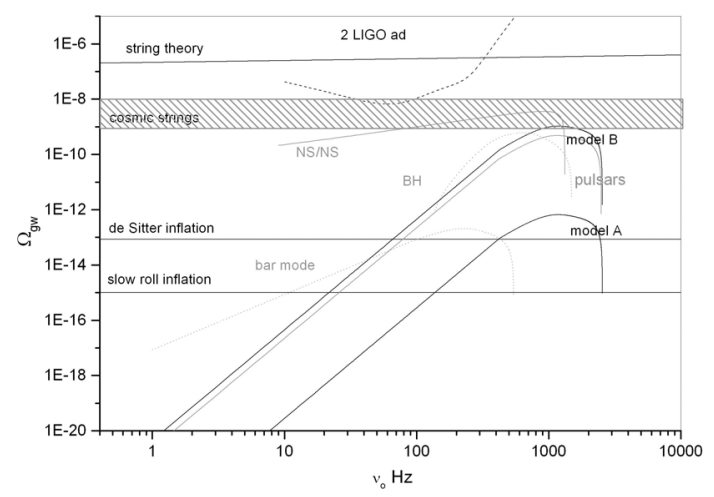

Fig. 7. Theoretical limits for some cosmological gravitational background models and present estimates for the magnetar contribution. Other astrophysical contributions (in grey) are plotted for comparison: continuous background (continuous line) such as NS/NS coalescence (Regimbau \& de Freitas Pacheco 2005) or rotating pulsars (updated from RP01b) and shot noise (dot line) such as $\mathrm{BH}$ ringdown and bar mode due to the Maclauren/Dedekind transition in young and fast rotating NS (updated from Regimbau 2001). The expected sensitivity resulting from the combination of two advanced interferometers is also shown for comparison.

high fields and to produce a considerable magnetic distortion $\left(g>10^{3}\right)$. The deformation of the star can be increased if the magnetic field has a geometry other than dipolar, since higher order multipoles will also contribute to the mass quadrupole. This is also true if the toroidal component is considered since its strength may be a factor of 10 higher than the poloidal component. Clearly these last possibilities require further work on NS structure when magnetic fields with complex geometries are considered.

The background from magnetars may not be detectable as a signal by the first generations of interferometers but it may behave as noise in the cosmological background frequency domain.

In Fig. 7, we have plotted some theoretical predictions for the CGB (Allen 1997; Maggiore 2000; Buonanno 2004), our estimates for the magnetar contribution along with other AGBs and the expected sensitivity of two correlated advanced interferometers. The amplification of vacuum fluctuations at the transition between the radiation and matter eras, first discussed by Grishchuk (1974, 1993) and Starobinsky (1979), is expected to produce a GW background whose spectrum and amplitude depend strongly on the fluctuation power spectrum developed during the early inflationary period.

The standard de Sitter inflation predicts a spectrum that decreases as $1 / f^{2}$ in the range $3 \times 10^{-18}-1 \times 10^{-16} \mathrm{~Hz}$ and then remains constant in a very large band up to frequencies of the order of $\mathrm{MHz}$. The Cobe experiment provides an upper bound of $\Omega_{\mathrm{gw}}=9 \times 10^{-14}$ (Maggiore 2000; Buonanno 2004) for the flat region, which is below the estimated background from magnetars in the frequency range $400-2500 \mathrm{~Hz}$ for model A and $70-2500 \mathrm{~Hz}$ for model $\mathrm{B}$. These predictions have been questioned recently by Grishchuk (2005). In the more realistic scenario of "slow roll down" inflation, the predicted amplitude is even lower, by almost two orders of magnitude. A more interesting case arises from the string cosmology since the expected amplitude is around $\Omega_{\mathrm{gw}}=10^{-7}$, well above the expected contribution from magnetars. However, other choices of the model parameters may lead to substantially lower amplitudes. Cosmic strings are also able to produce a CGB in the frequency range of the magnetar contribution, since the vibrations of these topological defects are sources of GW (Buonanno 2004). The spectrum is almost flat in a large frequency band $\left(10^{-8}-10^{-10}\right)$ with an upper limit for the amplitude of $10^{-9}-10^{-8}$ according to numerical simulations of a cosmic strings network that satisfy the bound imposed by the timing of pulsars. As it can be seen from Fig. 7, the magnetar contribution from model B is barely comparable to the predictions of this model at frequencies around $1 \mathrm{kHz}$. However, according to a recent study by Damour \& Vilenkin $(2000,2001,2005)$ the background from a network of cosmic strings at the end of Brane inflation may be not continuous but rather a collection of bursts emitted from cusps of oscillating string loops. In this case, it would be distinct in its statistical properties from the magnetar contribution.

\section{Conclusions}

In the present work, the contribution of magnetars to the stochastic background of gravitational waves was investigated, under the assumption that these highly magnetized stars are typical NS born in the high side tail of the initial field distribution (RP01a). Their geometric deformation is a consequence of such fields.

The statistical properties of the magnetar population such as the magnetic field and ellipticity distributions and their relative orientation were derived by simulations and then by supposing that the extra-galactic population has similar properties. Our new population synthesis, including new pulsars discovered by the Parkes multibeam survey, indicates that magnetars (neutron stars born with fields higher than $10^{14} \mathrm{G}$ ) constitute $8 \%$ of the total population.

Two models for the neutron star interior have been considered in our calculations: in the first (model A), the NS has a normal conducting interior, leading to deformation parameters $g$ in the range $1-20$. Here, the value $g=13$ was adopted (Konno et al. 2000). The second possibility (model B) considers that the NS develops a type I superconducting interior 
with a typical deformation parameter $g=520$ (Bonazzola \& Gourgoulhon 1996).

We found that magnetars produce a continuous background (duty cycle $\gg 1$ ) with a maximum around $1.2 \mathrm{kHz}$. This value is slightly dependent on the assumed maximum rotation velocity of the NS at birth, here taken equal to $1250 \mathrm{~Hz}$ (corresponding to a minimum initial rotation period of $0.8 \mathrm{~ms}$ ). The different cosmic star formation rates do not considerably affect the resulting density parameter $\Omega_{\mathrm{gw}}$, whose amplitude depends on the deformation parameter $g$, e.g., on the conducting properties of the NS interior. Amplitudes higher than the maximum found in this work, e.g., $\Omega_{\mathrm{gw}} \sim 10^{-9}$, could be expected if neutron stars have deconfined cores and a diquark condensate forms a second kind superconducting medium. However, estimates of the resulting deformation parameter under these conditions remain to be done. In spite of the maximum amplitude being inside the frequency band of ground based interferometers, the expected signal is well below the sensitivity of the first generation of detectors, but exceeds the upper bound derived from COBE data. Comparing our estimates to the most optimistic expectations of the gravitational background generated by some possible models, we find that the magnetar contribution does not overwhelm the relic emission expected from string cosmologies, but could be comparable around $1.0 \mathrm{kHz}$ to the limits estimated for the emission from oscillating cosmic strings. Above $70-120 \mathrm{~Hz}$ the magnetar contribution surpass the theoretical limits of the inflationary CGB, suggesting that searches should remain well below those frequencies. In spite of the maximum amplitude lying inside the frequency band of ground based interferometers, the expected signal is well below the sensitivity of the first generation of detectors.

Acknowledgements. We are grateful to L. Grishchuk and M. Maggiore for discussions during the preparation of this manuscript.

\section{References}

Andersson, N., \& Kokkotas, K. D. 2001, Int. J. Mod. Phys. D, 10, 381 Allen, B. 1997, Relativistic Gravitation and Gravitational Radiation, ed. J. A. Marck, \& J. P. Lasota (Cambridge University Press), 373 Bildsten, L. 1998, ApJ, 501, L89

Blaschke, D., Sedrakian, D. M., \& Shahabasyan, K. M. 1999, A\&A, 350,47

Bonazzola, S., \& Gourgoulhon, E. 1996, A\&A, 312, 675

Buonanno, A. 2004, [gr-qc/0303085]

Chandrasekhar, S., \& Fermi, E. 1953, ApJ, 118, 116

Christensen, N. 1997, PRD, 55, 448

Coccia, E., \& Fafone, V. 1997, Omnidirectional Gravitational Radiation Observatory, ed. W. F. Velloso, O. D. Aguiar, \& N. S. Magalhães (World Scientific), 113
Coward, D., Burman, R. R., \& Blair, D. 2001, MNRAS, 324, 1015

Damour, T., \& Vilenkin, A. 2000, Phys. Rev. Lett., 85, 3761

Damour, T., \& Vilenkin, A. 2001, PRD, 64, 064008

Damour, T., \& Vilenkin, A. 2005, PRD, 71, 063510

Damour, T., Iyer, B. R., \& Sathyaprakash, B. S. 2001, PRD, 63, 044023

de Bernardis, P., et al. 2000, Nature, 404, 995

de Freitas Pacheco, J. A. 1998, A\&A, 336, 397

de Freitas Pacheco, J. A. 2001, in Relativistic Aspects of Nuclear Physics, ed. T. Kodama, C. E. Aguiar, H.-T. Elze, F. Grassi, Y. Hama, \& G. Krein (World Scientific), 158

Duncan, R. C., \& Thomson, C. 1992, ApJ, 392, L29

Ferrari, V., Matarrese, S., \& Schneider, R. 1999, MNRAS, 303, 258

Ferraro, V. C. A. 1954, ApJ, 119, 407

Galtsov, D. V., \& Tsvetkov, V. P. 1984, Phys. Lett., 103A, 193

Grishchuk, L. P. 1974, Sov. Phys. JETP, 40, 409

Grishchuk, L. P. 1976, Sov. Phys. JETP Lett., 23, 293

Grishchuk, L. P. 1993, CQG, 10, 2449

Grishchuk, L. P. 2005, [gr-qc/0504018]

Grishchuk, L. P., Lipunov, V. M., Postnov, K. A., Prokhorov, M. E., \& Sathyaprakash, B. S. 2001, Physics-Uspekhi, 44, 1 1993, CQG, 10,2449

Hanany, S., Ade, P., Balbi, A., et al. 2000, ApJ, 545, L5

Heyl, J. S., \& Kulkarni, S. R. 1998, ApJ, 506, L61

Konno, K., Obata, T., \& Kojima, Y. 1999, A\&A, 352, 211

Konno, K., Obata, T., \& Kojima, Y. 2000, A\&A, 356, 234

Kouveliotou, C., Dieter, S., Strohmayer, T., et al. 1998, Nature, 393, 235

Kouveliotou, C., Strohmayer, T., Hurley, K., et al. 1999, ApJ, 510, L115

Maggiore, M. 2000, Phys. Rep., 331, 283

Marranghello, G. F., Vasconcellos, C. Z., \& de Freitas Pacheco, J. A. 2002, PRD, 66, 064020

Mereghetti, S. 1999, in The Neutron Star-Black Hole Connexion, NATO-ASI, 13 [arXiv: astro-ph/9911252]

Owen, B. J. 2005, Phys. Rev. Lett., 95, 211101

Palomba, C. 2001, A\&A, 367, 525

Porciani, C., \& Madau, P. 2001, ApJ, 548, 522

Regimbau, T. 2001, Ph.D. Thesis, University of Nice

Regimbau, T., \& de Freitas Pacheco, J. A. 2000, A\&A, 359, 242 (RP00)

Regimbau, T., \& de Freitas Pacheco, J. A. 2001a, A\&A, 374, 182 (RP01a)

Regimbau, T., \& de Freitas Pacheco, J. A. 2001b, A\&A, 376, 381 (RP01b)

Regimbau, T., \& de Freitas Pacheco, J. A. 2005, in preparation

Schmidt, B., Suntzeff, N. B., Phillips, M. M., et al. 1998, ApJ, 507, 46

Spergel, D. N., Verde, L., Peiris, H. V., et al. 2003, ApJS, 148, 175

Thorne, K. S. 1980, Rev. Mod. Phys., 52, 299

Ushomirsky, G., Cutler, C., \& Bildstel, L. 2000, MNRAS, 319, 902

Young, M. D., Manchester, R. N., \& Johnson, S. 1999, Nature, 400, 848

Zimmermann, M., \& Szedenits, E. 1979, PRD, 20, 351 